\title{
Equilibrium and Stability of Off-Axis Periodically Focused Particle Beams
}

\author{
J. S. Moraes, ${ }^{1,2, *}$ R. Pakter, ${ }^{1, \dagger}$ and F. B. Rizzato ${ }^{1, \dagger}$ \\ ${ }^{1}$ Instituto de Física, Universidade Federal do Rio Grande do Sul, Caixa Postal 15051, 91501-970 Porto Alegre, \\ Rio Grande do Sul, Brasil \\ ${ }^{2}$ Centro Universitário La Salle, Avenida Victor Barreto, 2288, 92010-000, Canoas, RS, Brasil
}

(Received 14 September 2004; published 7 December 2004)

\begin{abstract}
A general equation for the centroid motion of free, continuous, intense beams propagating off axis in solenoidal periodic focusing fields is derived. The centroid equation is found to be independent of the specific beam distribution and may exhibit unstable solutions. A new Vlasov equilibrium for off-axis beam propagation is also obtained. The properties of the equilibrium and the relevance of centroid motion to beam confinement are discussed.
\end{abstract}

DOI: 10.1103/PhysRevLett.93.244801

A fundamental understanding of the kinetic equilibrium and stability properties of certain systems is sought. These systems include periodically focused high-current, low emittance beams. The understanding we seek is crucial for a variety of applications. Among them are advanced particle accelerators and coherent radiation sources. For a long time, the Kapchinskij-Vladimirskij (KV) distribution [1] was the only Vlasov [2] equilibrium distribution known for the propagation of periodically focused intense particle beams. Equilibrium and stability analysis based on the KV beam have been critical to the development and understanding of the physics of intense beams [3-11]. More recently, it has been shown that the $\mathrm{KV}$ distribution can be generalized to allow for rigid beam rotation with respect to the Larmor frame in periodic solenoidal focusing fields [12]. Studies indicate that rotation may have an important role in particle beam stability [13].

In the derivation of these Vlasov equilibria it is always assumed that the beam is perfectly aligned with the symmetry axis of the focusing field $[1,6,12]$. Actually, this simplifying assumption is generally used in the analysis of intense beams [8] because the axis is an equilibrium for the beam centroid, and the equilibrium is stable if smooth-beam approximations are employed where the periodic fluctuations of the focusing field are averaged out [14]. In some cases, however, we may expect the onset of a parametric resonance involving the centroid motion and the focusing field oscillations. This would destabilize the centroid motion and heavily affect the overall beam dynamics. In such conditions the averaging procedure is no longer valid and a detailed description of the centroid dynamics becomes mandatory.

In this Letter, we derive from a kinetic VlasovMaxwell description a general equation for the centroid motion of free, continuous, intense beams propagating off axis in solenoidal periodic focusing fields. It is shown that the centroid obeys a Mathieu-type equation. The equation is independent of the specific beam distribution and becomes unstable whenever the oscillatory frequency of the centroid, which is related to the rms focusing field
PACS numbers: 41.85.Ja, 05.45.-a

strength per lattice, is commensurable with the focusing field periodicity itself. In the particular case of a uniform beam density around the beam centroid, we show that there exists a self-consistent Vlasov equilibrium distribution for the beam dynamics. The beam envelope that determines the outer radius of the equilibrium beam around the centroid is shown to obey the familiar envelope equation $[6,8,15,16]$, being independent of the centroid motion. An example of the Vlasov equilibrium is discussed in detail to show the possibility of finding beam solutions for which the extensively studied envelope equation $[4,5,9-11,17]$ is stable, whereas the centroid motion is unstable, revealing the importance of the centroid motion to overall beam confinement properties.

We consider a free, continuous charged-particle beam propagating with average axial velocity $\beta_{b} c \hat{\mathbf{e}}_{z}$ through a periodic solenoidal focusing magnetic field described by

$$
\mathbf{B}(\mathbf{r}, s)=B_{z}(s) \hat{\mathbf{e}}_{z}-\frac{r}{2} B_{z}^{\prime}(s) \hat{\mathbf{e}}_{r},
$$

where $\mathbf{r}=x \hat{\mathbf{e}}_{x}+y \hat{\mathbf{e}}_{y}, r=\left(x^{2}+y^{2}\right)^{1 / 2}$ is the radial distance from the field symmetry axis, $s=z=\beta_{b} c t$ is the axial coordinate, $B_{z}(s+S)=B_{z}(s)$ is the magnetic field on the axis, the prime denotes derivative with respect to $s$, $c$ is the speed of light in vacuo, and $S$ is the periodicity length of the magnetic focusing field. Since we are dealing with solenoidal focusing, it is convenient to work in the Larmor frame of reference [8], which rotates with respect to the laboratory frame with angular velocity $\Omega_{L}(s)=q B_{z}(s) / 2 \gamma_{b} m c$, where $q, \quad m$, and $\gamma_{b}=$ $\left(1-\beta_{b}^{2}\right)^{-1 / 2}$ are, respectively, the charge, mass, and relativistic factor of the beam particles. The Larmor frame is used throughout the Letter, such that $\hat{\mathbf{e}}_{x}$ and $\hat{\mathbf{e}}_{y}$ are assumed to be unit vectors along the rotating axes. In the paraxial approximation, the beam distribution function $f_{b}(\mathbf{r}, \mathbf{v}, s)$ evolves according to the Vlasov-Maxwell system [8]

$$
\frac{\partial f_{b}}{\partial s}+\mathbf{v} \cdot \nabla f_{b}+\left(-\kappa_{z} \mathbf{r}-\nabla \psi\right) \cdot \nabla_{\mathbf{v}} f_{b}=0,
$$




$$
\begin{gathered}
\nabla^{2} \psi=-\left(2 \pi K / N_{b}\right) n_{b}(\mathbf{r}, s), \\
n_{b}=\int f_{b} d \mathbf{v},
\end{gathered}
$$

where $n_{b}(\mathbf{r}, s)$ is the beam density profile, $\kappa_{z}(s)=$ $q^{2} B_{z}^{2}(s) / 4 \gamma_{b}^{2} \beta_{b}^{2} m^{2} c^{4}$ is the focusing field parameter, $K=$ $2 q^{2} N_{b} / \gamma_{b}^{3} \beta_{b}^{2} m c^{2}$ is the beam perveance, $N_{b}=$ $\int f_{b} d \mathbf{r} d \mathbf{v}=$ const is the number of particles per unit axial length, and $\mathbf{v} \equiv \mathbf{r}^{\prime}$ [18]. The Vlasov equation (2) expresses probability conservation in our model. In Eqs. (2)-(4), $\psi$ is a normalized potential that incorporates both selfelectric and self-magnetic fields, $\mathbf{E}^{s}$ and $\mathbf{B}^{s}$. A detailed derivation [8], shows that $\psi$ is related to the self-scalar and self-vector potentials by $\phi^{s}=\beta_{b}^{-1} A_{z}^{s}=$ $\gamma_{b}^{3} m \beta_{b}^{2} c^{2} \psi(\mathbf{r}, s) / q, \quad$ where $\quad \mathbf{A}^{s}(\mathbf{r}, s)=A_{z}^{s}(\mathbf{r}, s) \hat{\mathbf{e}}_{z}$, $\mathbf{E}^{s}(\mathbf{r}, s)=-\nabla \phi^{s}(\mathbf{r}, s)$, and $\mathbf{B}^{s}(\mathbf{r}, s)=\nabla \times \mathbf{A}^{s}(\mathbf{r}, s)$.

Our first task here is to determine the evolution of the beam centroid located at

$$
\overline{\mathbf{r}}(s) \equiv N_{b}^{-1} \int \mathbf{r} f_{b}(\mathbf{r}, \mathbf{v}, s) d \mathbf{r} d \mathbf{v} .
$$

In order to do that one multiplies Eq. (2) by $\mathbf{r}$ and integrates over phase space to get

$$
\overline{\mathbf{r}}^{\prime}=\overline{\mathbf{v}}
$$

where $\overline{\mathbf{v}} \equiv N_{b}^{-1} \int \mathbf{v} f d \mathbf{r} d \mathbf{v}$. If one now multiplies Eq. (2) by $\mathbf{v}$ and integrates over phase space, one obtains

$$
\overline{\mathbf{v}}^{\prime}=-\kappa_{z} \overline{\mathbf{r}}-\overline{\nabla \psi},
$$

where $\overline{\nabla \psi} \equiv N_{b}^{-1} \int \nabla \psi f d \mathbf{r} d \mathbf{v}$ is obtained by integration by parts of the $\nabla_{\mathbf{v}}$ term in velocity space. Using Eqs. (3) and (4) we can rewrite $\overline{\nabla \psi}$ as

$$
\overline{\nabla \psi}=(2 \pi K)^{-1} \int \nabla \psi \nabla^{2} \psi d \mathbf{r} .
$$

Then we note that the integrand of Eq. (8) can be cast into the more suitable form

$$
\nabla \psi \nabla^{2} \psi=\nabla \cdot\left[\nabla \psi \nabla \psi-\mathbf{I}(\nabla \psi)^{2} / 2\right],
$$

where the unit dyadic $\mathbf{I}$ reads $\mathbf{I} \equiv \hat{\mathbf{e}}_{x} \hat{\mathbf{e}}_{x}+\hat{\mathbf{e}}_{y} \hat{\mathbf{e}}_{y}$. Now, employing Gauss theorem we obtain

$$
\overline{\nabla \psi}=(2 \pi K)^{-1} \oint \hat{\mathbf{e}}_{n} \cdot\left[\nabla \psi \nabla \psi-\mathbf{I}(\nabla \psi)^{2} / 2\right] d A=0,
$$

because $\nabla \psi \rightarrow 0$ as $r \rightarrow \infty$ for beams in free space. In Eq. (10), $d A$ and $\hat{\mathbf{e}}_{n}$ are, respectively, the boundary differential element and the unit vector normal to the boundary of integration located at $r \rightarrow \infty$. In fact, the result $\overline{\nabla \psi}=0$ is expected based on the action-reaction law; since $-\nabla \psi$ corresponds to the self-force exerted on the beam particles by themselves, its average throughout the beam distribution has to vanish due to the pairwise structure of the interparticle electromagnetic interaction. Using Eqs. (7) and (10) in Eq. (6), we finally obtain the centroid equation of motion

$$
\overline{\mathbf{r}}^{\prime \prime}+\kappa_{z}(s) \overline{\mathbf{r}}=0 .
$$

Let us stress that we have not made any assumption on the particular form of the beam distribution function so far. Thus, the centroid equation above is always valid as long as the beam evolves according to the Vlasov-Maxwell system, Eqs. (2)-(4). In the laboratory frame, combined to the oscillatory motion described by Eq. (11) the centroid also rotates with angular velocity $\Omega_{L}(s)$ around the center $r=0$. Taking into account that $\kappa_{z}(s)$ is periodic, Eq. (11) is of the Mathieu type, which describes a periodically forced harmonic oscillator. This equation is known to present unstable solutions related to parametric resonances in the $\overline{\mathbf{r}}$ motion. If we conveniently write the average of $\kappa_{z}(s)$ over one lattice period as $(1 / S) \times$ $\int_{0}^{S} \kappa_{z}(s) d s \equiv \sigma_{0}^{2} / S^{2}$, where $\sigma_{0}$ is a dimensionless parameter proportional to the rms focusing field measuring the vacuum phase advance in the small field, smoothbeam approximation, the instabilities in the centroid motion are expected when one approaches $\sigma_{0} \sim n \pi$; this condition corresponds to parametric resonances between the oscillation periodicity of $\overline{\mathbf{r}}$ in the average (rms) focusing field and the periodicity of the focusing field itself. Depending on the exact profile of $\kappa_{z}(s)$ the size of the unstable regions surrounding $\sigma_{0} \sim n \pi$ vary significantly. If the aim is beam confinement, these regions are to be avoided.

It is worth mentioning that although Eq. (11) is strictly valid for free beams only, it is expected to provide a good description of the centroid motion in bounded systems if the beam is nearly symmetric and is not excessively displaced from a pipe center located at $r=0$. The reason is because in this case $\nabla \psi= \pm \hat{\mathbf{e}}_{n}|\nabla \psi|$ at the pipe walls, where $\hat{\mathbf{e}}_{n}$ is now the unit vector normal to the wall, and the surface integral in Eq. (10), performed along the boundary, still vanishes since $|\nabla \psi|$ is approximately constant there. Note also that the presence of a pipe would generally not suppress the centroid instabilities discussed in connection with Eq. (11); in fact, it would even enhance it because the image charges induced are of opposite sign, attracting the beam to the wall.

Our next task is to show that we can construct a Vlasov equilibrium for off-axis beam transport. In particular, we assume a beam with a uniform radial density distributed around a center located at $\mathbf{r}_{0}(s)=x_{0}(s) \hat{\mathbf{e}}_{x}+y_{0}(s) \hat{\mathbf{e}}_{y}$, i.e.,

$$
n_{b}(\mathbf{r}, s)= \begin{cases}N_{b} / \pi r_{b}^{2}(s), & r_{\delta}<r_{b}(s) \\ 0, & r_{\delta}>r_{b}(s)\end{cases}
$$

where $r_{b}(s)$ is the equilibrium beam envelope and $\mathbf{r}_{\delta} \equiv$ $\mathbf{r}-\mathbf{r}_{0}$. A schematic of the beam distribution of Eq. (12) and corresponding vectors is shown in Fig. 1. For such a beam we can easily recognize $\mathbf{r}_{0}(s)$ as being the centroid coordinate. According to what was shown previously, its evolution must then obey

$$
\mathbf{r}_{0}^{\prime \prime}+\kappa_{z}(s) \mathbf{r}_{0}=0
$$




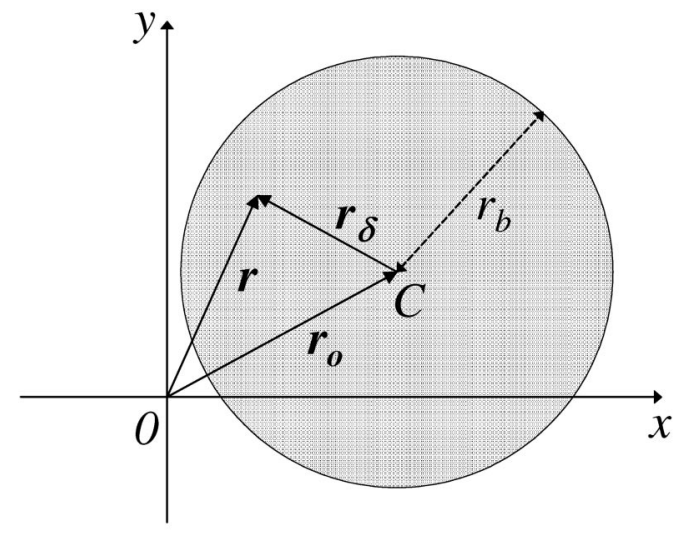

FIG. 1. Schematic of the beam distribution of Eq. (12) and corresponding vectors. $C$ corresponds to the centroid position.

Using the prescribed $n_{b}(\mathbf{r}, s)$ in Eq. (3) we find for the normalized self-potential

$$
\psi(\mathbf{r}, s)=-K r_{\delta}^{2} / 2 r_{b}^{2}(s)
$$

in the beam interior $\left(r_{\delta}<r_{b}\right)$. Therefore, a singleparticle of the beam located at $\mathbf{r}(s)$ subjected to the external focusing field force $-\kappa_{z}(s) \mathbf{r}$ and the self-field force $-\nabla \psi(\mathbf{r}, s)$ will evolve according to

$$
\mathbf{r}^{\prime \prime}+\kappa_{z} \mathbf{r}-\left(K / r_{b}^{2}\right) \mathbf{r}_{\delta}=0 .
$$

If we now subtract Eq. (13) from Eq. (15) we obtain

$$
\mathbf{r}_{\delta}^{\prime \prime}+\kappa_{z} \mathbf{r}_{\delta}-\left(K / r_{b}^{2}\right) \mathbf{r}_{\delta}=0,
$$

which describes the motion of the beam particle with respect to the center $\mathbf{r}_{0}$. Equation (16) can be solved with known techniques of physics of beams $[6,8]$. Considering the motion along the $x$ axis, we write $x_{\delta}=$ $A_{x_{\delta}} w(s) \sin \left[\int^{s} \zeta(s) d s+\zeta_{x_{\delta} 0}\right]$ with $A_{x_{\delta}}$ and $\zeta_{x_{\delta} 0}$ constants. Substituting this expression into Eq. (16) we obtain

$$
w^{\prime \prime}+\kappa(s) w=w^{-3},
$$

$\zeta(s)=w^{-2}(s)$, where $\kappa(s) \equiv \kappa_{z}(s)-K / r_{b}^{2}(s)$, and the constant of motion $A_{x_{\delta}}$ can be expressed in the form

$$
A_{x_{\delta}}^{2}=\left(x_{\delta} / w\right)^{2}+\left(w x_{\delta}^{\prime}-w^{\prime} x_{\delta}\right)^{2} .
$$

Performing an equivalent calculation for the motion along the $y$ axis, one shows that $A_{y_{\delta}}$ given by

$$
A_{y_{\delta}}^{2}=\left(y_{\delta} / w\right)^{2}+\left(w y_{\delta}^{\prime}-w^{\prime} y_{\delta}\right)^{2}
$$

is also a constant of motion. From Eq. (16) one sees that all the forces are central with respect to the centroid $\mathbf{r}_{0}$. Thus, one readily demonstrates that the canonical angular momentum $P_{\Theta \delta}$ given by

$$
P_{\Theta \delta}=x_{\delta} y_{\delta}^{\prime}-y_{\delta} x_{\delta}^{\prime}
$$

is a constant of motion as well. Because $A_{x_{\delta}}^{2}, A_{y_{\delta}}^{2}$, and $P_{\Theta \delta}$ are exact single-particle constants of motion, a possible choice of Vlasov equilibrium distribution function is

$$
\begin{aligned}
f_{b}^{E Q}(\mathbf{r}, \mathbf{v}, s)= & \frac{N_{b}}{\pi^{2} \epsilon_{T}} \delta\left[A_{x_{\delta}}^{2}+A_{y_{\delta}}^{2}-2 \omega_{b} P_{\Theta \delta}\right. \\
& \left.-\left(1-\omega_{b}^{2}\right) \boldsymbol{\epsilon}_{T}\right],
\end{aligned}
$$

where $d f_{b}^{E Q} / d s=0, \epsilon_{T}=$ const is an effective emittance, and the rotation parameter $\omega_{b}=$ const is in the range $-1<\omega_{b}<1$ for radially confined beams. Using $f_{b}^{E Q}$ in Eq. (4), it is readily shown that the uniform density profile centered at $\mathbf{r}_{0}$ of Eq. (12) is consistently obtained, provided $r_{b}(s)=\epsilon_{T}^{1 / 2} w(s)$. Hence, $r_{b}(s)$ obeys the familiar envelope equation

$$
r_{b}^{\prime \prime}+\kappa_{z}(s) r_{b}-\frac{K}{r_{b}}-\frac{\epsilon_{T}^{2}}{r_{b}^{3}}=0 .
$$

Performing the appropriate averages over the equilibrium distribution, Eq. (21), we can show that the beam rigidly rotates around its centroid $\mathbf{r}_{0}(s)$ with angular velocity $\Omega_{b \delta}(s)=\omega_{b} \epsilon_{T} \beta_{b} c / r_{b}^{2}(s)$, which shows that $\omega_{b}$ is related to the differential rotation in the Larmor frame. Also, the rms emittance calculated with respect to the centroid position given by

$$
\epsilon_{\chi} \equiv 4\left[\left\langle\chi^{2}\right\rangle\left\langle\chi^{\prime 2}\right\rangle-\left\langle\chi \chi^{\prime}\right\rangle^{2}\right]^{1 / 2}
$$

is equal to the effective emittance parameter $\epsilon_{T} \cdot \chi=$ $x_{\delta}, y_{\delta}$, and the brackets indicate averages over the beam distribution. One thus sees that a Vlasov equilibrium distribution can be formed for which the beam envelope obeys Eq. (22) with constant emittance even when the centroid moves off axis, $\mathbf{r}_{0} \neq 0$, following the dynamics dictated by Eq. (13). We refer to this equilibrium as a periodically focused off-axis Vlasov equilibrium. Let us call attention to the interesting fact that the centroid motion and the envelope dynamics are uncoupled in this case. In other words, centroid dynamics does not affect the known stability results for the envelope dynamics $[4,5,9,11,17]$ and is not affected by the latter as well. One should keep in mind that for good beam confinement both centroid and envelope have to be stable.

We now illustrate our results with an example of periodically focused off-axis Vlasov equilibrium. We consider a particular set of parameters for which the envelope Eq. (22) is known to be stable, whereas the centroid motion of Eq. (13) was found to be unstable. We investigate beam transport with the aid of selfconsistent numerical simulations, where a large number $N_{b}=8000$ of macroparticles interact via pairwise electromagnetic interactions [10]. In the simulation we used $S K / \epsilon_{T}=5.0$ and $S^{2} \kappa_{z}(s)=\sigma_{0}^{2}[1+\cos (2 \pi s / S)]$, with $\sigma_{0}=155^{\circ}$, over 20 lattice periods. The macroparticles were launched at $s=0$ according to the equilibrium distribution, Eq. (21), with $\omega_{b}=0, \mathbf{r}_{0}=0=\mathbf{r}_{0}{ }^{\prime}$, and $r_{b}$ corresponding to the matched solution with $r_{b}(s+S)=$ $r_{b}(s)$ of the envelope Eq. (22). The finite number of macroparticles in the initial condition acts as a seed for 


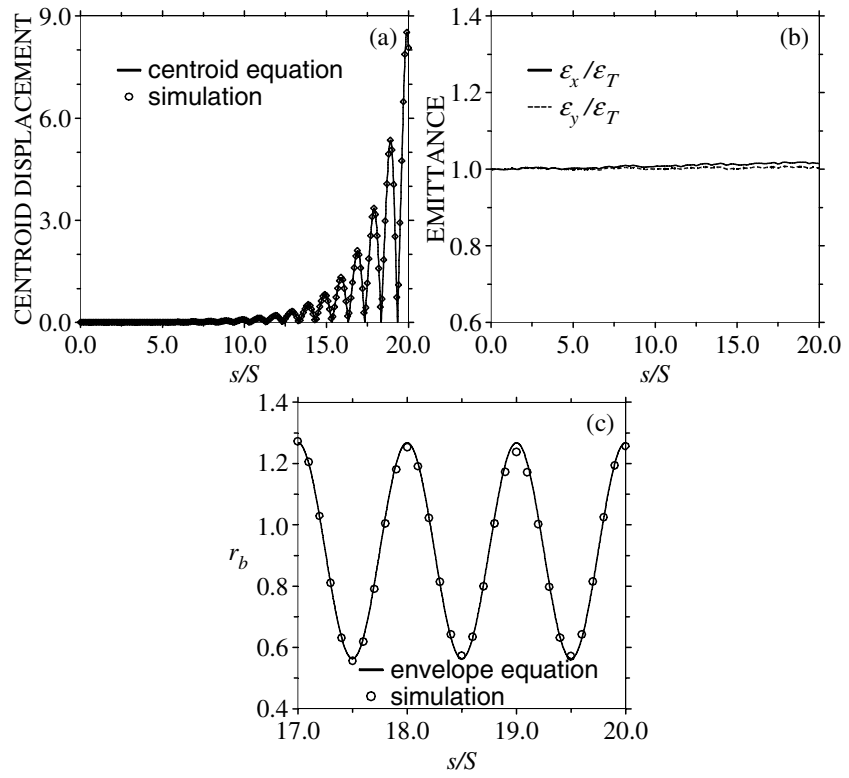

FIG. 2. Multiparticle self-consistent simulation results. (a) The centroid motion, (b) rms emittance, and (c) the envelope dynamics. Centroid displacement and envelope are normalized to $\left(S \epsilon_{T}\right)^{1 / 2}$.

any possible instability to develop. Simulation results are presented in Fig. 2. The evolution of the centroid displacement $r_{0} \equiv\left|\mathbf{r}_{0}\right|$ calculated from the macroparticles positions $\mathbf{r}$ as $\mathbf{r}_{0}=\langle\mathbf{r}\rangle$, where the brackets indicate average over macroparticles, is shown in Fig. 2(a) (circles). It reveals that the centroid motion develops the typical exponential growth of unstable dynamics that agrees with the fact that the set of parameters considered leads to an unstable solution of Eq. (13). The solid line corresponds to the solution obtained from Eq. (13). Despite the centroid instability, the beam equilibrium distribution is preserved as verified in Fig. 2(b) that shows that rms emittance is well conserved as the beam evolves. The rms emittance is calculated according to Eq. (23), considering averages over macroparticles. Finally, Fig. 2(c) compares the envelope obtained from the envelope Eq. (22) with that obtained from the simulation, $r_{b}=$ $\left[2\left\langle\left(\mathbf{r}-\mathbf{r}_{0}\right)^{2}\right\rangle\right]^{1 / 2}$, for the last three periods of the focusing channel. The perfect agreement proves once more the preservation of the equilibrium distribution. Moreover, we see that in spite of the unstable centroid the envelope is stable, as predicted.

To conclude, based on kinetic grounds we have derived a general equation for the centroid motion of free, continuous, intense beams propagating off axis in solenoidal periodic focusing fields. It was shown that the centroid equation is independent of the specific beam distribution and may exhibit unstable solutions. In the particular case of a uniform beam density around the beam centroid, we have shown the existence of a periodically focused offaxis Vlasov equilibrium distribution describing a beam that rigidly rotates with a prescribed angular velocity around a moving centroid. The beam envelope around the centroid was shown to obey the familiar envelope equation, being independent of the centroid motion. An example of periodically focused off-axis Vlasov equilibrium was discussed in detail to show the possibility of finding beam solutions for which the envelope equation is stable, whereas the centroid motion is unstable, revealing the importance of centroid motion to the overall beam confinement properties. The purpose of the present theory is to help understanding results of existing and proposed experiments in particle acceleration and high power microwave sources, where off-axis dynamics is of relevance [14].

We acknowledge partial support from $\mathrm{CNPq}$, Brazil.

*Email address: jsmoraes@if.ufrgs.br

†Email address: pakter@if.ufrgs.br

*Email address: rizzato@if.ufrgs.br

[1] I. M. Kapchinskij and V.V. Vladimirskij, in Proceedings of the International Conference on High Energy Accelerators (CERN, Geneva, 1959), p. 274.

[2] Y.L. Klimontovich, The Statistical Theory of NonEquilibrium Processes in Plasma (Pergamon, Oxford, 1967).

[3] I. Hofmann, L. J. Laslett, L. Smith, and I. Haber, Part. Accel. 13, 145 (1983).

[4] C. J. Struckmeier and M. Reiser, Part. Accel. 14, 227 (1984).

[5] C. Chen and R. C. Davidson, Phys. Rev. Lett. 72, 2195 (1994).

[6] C. Chen and R. C. Davidson, Phys. Rev. E 49, 5679 (1994).

[7] R. L. Gluckstern, W.-H. Cheng, and H. Ye, Phys. Rev. Lett. 75, 2835 (1995).

[8] R. C. Davidson and H. Qin, Physics of Intense Charged Particle Beams in High Energy Accelerators (World Scientific, Singapore, 2001).

[9] R. Pakter and F. B. Rizzato, Phys. Rev. Lett. 87, 044801 (2001).

[10] R. Pakter and F. B. Rizzato, Phys. Rev. E 65, 056503 (2002).

[11] S. M. Lund and B. Bukh, Phys. Rev. ST Accel. Beams 7, 024801 (2004).

[12] C. Chen, R. Pakter, and R. C. Davidson, Phys. Rev. Lett. 79, 225 (1997).

[13] C. Chen, R. Pakter, and R. C. Davidson, Phys. Plasmas 6, 3647 (1999).

[14] M. Hess and C. Chen, Phys. Plasmas 7, 5206 (2000); Phys. Lett. A 295, 305 (2002).

[15] P. M. Lapostolle, IEEE Trans. Nucl. Sci. NS-18, 1101 (1971).

[16] F. J. Sacherer, IEEE Trans. Nucl. Sci. NS-18, 1105 (1971).

[17] J. S. Moraes, F. B. Rizzato, and R. Pakter, Phys. Plasmas 10, 4811 (2003).

[18] A note on the notation used. The vector derivatives with respect to $s$ in this Letter represent derivations directly in the Larmor frame, where $\hat{\mathbf{e}}_{x}$ and $\hat{\mathbf{e}}_{y}$ are considered fixed unit vectors. 